\title{
AGE-modified basement membrane cooperates with Endo180 to promote epithelial cell invasiveness and decrease prostate cancer survival
}

Mercedes Rodriguez-Teja, ',6 Julian H Gronau,'\# Claudia Breit,'\# Yu Zhi Zhang,' Ai Minamidate,'

Matthew P Caley,' Afshan McCarthy, ${ }^{3}$ Thomas R Cox, Janine T Erler, ${ }^{7}$ Luke Gaughan, ${ }^{7}$ Steven Darby,

Craig Robson, ${ }^{4}$ Francesco Mauri, ${ }^{2}$ Jonathan Waxman' and Justin Sturge ${ }^{1,5 *}$

I Department of Surgery and Cancer, Imperial College London, UK

2 Department of Medicine, Imperial College London, UK

3 Section of Cell and Molecular Biology, Institute of Cancer Research, London, UK

4 Northern Institute for Cancer Research, North Terrace, Newcastle University Medical School, UK

5 School of Biological, Biomedical and Environmental Sciences, University of Hull, UK

6 Departamento de Genética, Facultad de Medicina, Universidad de la República, Montevideo, Uruguay

7 Biotech Research and Innovation Centre, University of Copenhagen, Denmark

*Correspondence to: J Sturge, School of Biological, Biomedical and Environmental Sciences, University of Hull, Cottingham Road, Hull HU6 7RX, UK. E-mail: j.sturge@hull.ac.uk

\#These authors contributed equally to this study.

\begin{abstract}
Biomechanical strain imposed by age-related thickening of the basal lamina and augmented tissue stiffness in the prostate gland coincides with increased cancer risk. Here we hypothesized that the structural alterations in the basal lamina associated with age can induce mechanotransduction pathways in prostate epithelial cells (PECs) to promote invasiveness and cancer progression. To demonstrate this, we developed a 3D model of PEC acini in which thickening and stiffening of basal lamina matrix was induced by advanced glycation end-product (AGE)-dependent non-enzymatic crosslinking of its major components, collagen IV and laminin. We used this model to demonstrate that antibody targeted blockade of CTLD2, the second of eight C-type lectin-like domains in Endo180 (CD280, CLEC13E, KIAA0709, MRC2, TEM9, uPARAP) that can recognize glycosylated collagens, reversed actinomyosin-based contractility [myosin-light chain-2 (MLC2) phosphorylation], loss of cell polarity, loss of cell-cell junctions, luminal infiltration and basal invasion induced by AGE-modified basal lamina matrix in PEC acini. Our in vitro results were concordant with luminal occlusion of acini in the prostate glands of adult Endo $180^{\Delta \mathrm{E} \times 2-6 / \Delta \mathrm{E} \times 2-6}$ mice, with constitutively exposed CTLD2 and decreased survival of men with early (non-invasive) prostate cancer with high epithelial Endo180 expression and levels of AGE. These findings indicate that AGE-dependent modification of the basal lamina induces invasive behaviour in non-transformed PECs via a molecular mechanism linked to cancer progression. This study provides a rationale for targeting CTLD2 in Endo180 in prostate cancer and other pathologies in which increased basal lamina thickness and tissue stiffness are driving factors.

Copyright @ 2014 Pathological Society of Great Britain and Ireland. Published by John Wiley \& Sons, Ltd.
\end{abstract}

Keywords: advanced glycation endproducts; ageing; basement membrane; cell contractility; collagen crosslinking; C-type lectin domain; epithelium; invasion; matrix stiffness; prostate cancer

Received 12 September 2014; Revised 5 November 2014; Accepted 8 November 2014

No conflicts of interest were declared.

The copyright line for this article was changed on 13 July 2015 after original online publication.

\section{Introduction}

Structural changes in the basal lamina during ageing include altered matrix composition, organization and progressive thickening [1,2]. The extent of these alterations is organ-specific and exacerbated by metabolic or endocrine disorders [3-5]. Age-related reorganization of the basement membrane in prostate gland acini was reported four decades ago and indicated that its thickness can increase from $400-700 \AA$ in younger (4-6 months) mice to $3000 \AA$ in older (29-36 months) mice [3]. Given that progressive prostate enlargement and stiffening occurs during the common age-related and metabolic condition benign prostate hyperplasia (BPH) [6], we proposed that this highly tensile environment can alter prostate epithelial cell (PEC) behaviour and promote tumour progression. 
Endo180 is a multiple C-type lectin-like domain (CTLD) receptor that binds to extracellular collagens via its fibronectin type II (FNII) domain [7] and glycosylated forms of collagen via its second CTLD, CTLD2 [8]. Endo180 is a highly sensitive and specific serum marker of metastatic disease in breast cancer [9], and its expression correlates with Gleason score and overall survival in prostate cancer [10,11]. Endo180-dependent prostate cancer progression involves its functional switch from suppressor to promoter of epithelial-mesenchymal transition (EMT) upon its disassociation from CD147 (EMMPRIN, Basigin) and relocalization from the plasma membrane to endosomes in PEC acini [11]. Given that endosomal Endo180 enhances myosin-light chain-2 (MLC2) phosphorylation and cell migration [12], we postulated that the basal lamina stiffness triggers Endo180-dependent PEC invasiveness in the ageing prostate gland.

\section{Materials and methods}

\section{Reconstituted basement membrane modification}

An adapted protocol [13] was used to crosslink Cultrex ${ }^{\circledR}$ Culture Matrix ${ }^{\mathrm{TM}}$ reduced growth factor-reconstituted basement membrane (rBM) extract (3445-005-01, Trevigen $\left.{ }^{\circledR}\right)$. Eight-well chamber slides were coated with $30 \mu \mathrm{l} 100 \%$ v/v Cultrex ${ }^{\circledR}$ rBM extract and incubated at $37^{\circ} \mathrm{C}$ for $30 \mathrm{~min}$. The resultant rBM gels were incubated at $37^{\circ} \mathrm{C}$ for 6 or $14 \mathrm{~h}$ with phosphate-buffered saline (PBS) alone or PBS containing $50 \mathrm{mM}$ glycolaldehyde (G6805, Sigma-Aldrich) in $0.2 \mathrm{M}$ phosphate buffer, $\mathrm{pH} 7.8$, followed by quenching with $1 \mathrm{M}$ glycine ethyl ester hydrochloride (50060, Sigma-Aldrich); 50 mM sodium cyanoborohydride (71435, Sigma-Aldrich) and $250 \mathrm{~mm}$ aminoguanidine hydrochloride (396494, Sigma-Aldrich) were respectively used to reduce Schiff bases and quench reactive aldehydes, preventing AGE formation. $\mathrm{rBM}$ gels were washed with PBS (5 min, five times), incubated in $\mathrm{PBS}$ at $37^{\circ} \mathrm{C}(16 \mathrm{~h})$ and washed with PBS (5 min, three times). For analysis, rBM gels were centrifuged at high speed $(5 \mathrm{~min})$, resuspended in $500 \mathrm{ml} \mathrm{70 \%} \mathrm{v/v} \mathrm{formic} \mathrm{acid} \mathrm{and} 20 \mathrm{mg} / \mathrm{ml}$ cyanogen bromide (C91492, Sigma-Aldrich) in $2 \mathrm{~g} / \mathrm{ml}$ acetonitrile (271004, Sigma-Aldrich), incubated at room temperature $(16 \mathrm{~h})$ and dialysed against water. Fluorophore production associated with AGE generation was determined (excitation wavelength $=370 \mathrm{~nm}$; absorbance wavelength $=440 \mathrm{~nm}$; PHERAstar Plus spectrophotometer, BMG Labtech). Corresponding rBM gel samples were resolved on $12 \% \mathrm{v} / \mathrm{v}$ SDS-PAGE gels (reducing conditions) and visualized (PageSilver ${ }^{\mathrm{TM}}$, Fermentas). For microscopic analysis of collagen IV and laminin fibres, eight-well chamber slides containing rBM gels were washed with PBS, fixed [4\% w/v paraformaldehyde (PFA) diluted in PBS, $30 \mathrm{~min}$ ], quenched (100 mM glycine in PBS; $10 \mathrm{~min}$, three times) and incubated at room temperature $(1 \mathrm{~h})$ in $1 \% \mathrm{w} / \mathrm{v}$ bovine serum albumin (BSA) and $2 \% \mathrm{v} / \mathrm{v}$ fetal calf serum (FCS) diluted in PBS, before immunostaining with rabbit anti-human collagen IV polyclonal antibody (pAb; R1041, Acris Antibodies) and rabbit anti-human laminin $\mathrm{pAb}$ (sc-5583, Santa Cruz). Samples were fixed and quenched (see above), washed with PBS (5 min, twice), mounted in Vectashield ${ }^{\circledR}$ and analysed by confocal microscopy (Zeiss Confocal Axiovert 200M operated by LSM510 META software). For rheometry, $8 \mathrm{~mm}$ diameter $\times 1 \mathrm{~mm}$ thick rBM gels were analysed at $21^{\circ} \mathrm{C}$ with an ARG2-controlled strain rotational rheometer (TA Instruments), using $8 \mathrm{~mm}$ parallel plate serrated geometry, $1-3 \%$ strain and fixed frequency oscillation of $1 \mathrm{~Hz}$. Gels displayed minimal frequency dependence and a linear viscoelastic response at these settings. The elastic modulus $(E)$ was determined from the shear storage modulus $\left(G^{\prime}\right)$, using the equation $E=2 \times G^{\prime} \times(1+v)$, where $v=$ Poisson's ratio $(0.5)$.

\section{Acinar morphogenesis and analysis}

RWPE-1 cells were obtained from the American Type Culture Collection, where they were verified by short tandem repeat profiling and phenotypic analysis. Cells were tested for mycoplasma every 3 months (Venor ${ }^{\mathrm{TM}} \mathrm{GeM}$ ) and used up to 10 passages for experiments. RWPE-1 cells were grown in complete keratinocyte serum-free medium (17005-075, Invitrogen). A modified method [14] was used to generate PEC acini. RWPE- 1 cells were seeded $\left(0.5 \times 10^{4}\right.$ cells $\left./ \mathrm{cm}^{2}\right)$ on PBS-treated (native) or glycolaldehyde-treated (stiff) rBM gels, overlaid with complete keratinocyte serum-free medium containing $2 \% \mathrm{v} / \mathrm{v} \mathrm{rBM}$, and cultured in a $5 \% \mathrm{v} / \mathrm{v} \mathrm{CO}_{2}$ incubator at $37^{\circ} \mathrm{C}$ (6 days) and mouse anti-human Endo180 $\mathrm{mAb}$ clone A5/158 [15] or non-specific isotype control IgG antibody (ab37355-1, Abcam), both at $10 \mu \mathrm{g} / \mathrm{ml}$, included where indicated and replaced every $24 \mathrm{~h}$. Three blinded scorers calculated the shape distribution of the acini prior to fixation, using an arbitrary system to score acini as round (smooth basal interface), semi-polygonal (irregular basal interface) or polygonal (protrusive basal interface). Relative acinar size and luminal area were calculated from confocal microscopy images of acini immunostained with phalloidin AlexaFluor ${ }^{\circledR} 488$ (A12379, Invitrogen) and TO-PRO ${ }^{\circledR}$-3-iodide nuclear counterstain (T3605, Sigma-Aldrich), using ImageJ $\mathrm{v} 1.44 \mathrm{p}$ algorithms. Acinar and luminal areas were calculated in pixels and values normalized against corresponding untreated samples (set at 100\%). The percentage of acini with $>1$ basal protrusions was quantified from epifluorescent images of acini stained with 4',6-diamidino-2-phenylindole (DAPI; D3571, Sigma-Aldrich). The percentages (mean $\pm \mathrm{SD}$ ) were quantified (50 acini/treatment) for three independent experimental repeats.

\section{Immunofluorescent staining}

RWPE-1 acini grown in eight-well chamber slides were washed in $\mathrm{PBS}+\left(0.1 \mathrm{mM} \mathrm{CaCl} 2\right.$ and $0.5 \mathrm{mM} \mathrm{MgCl}_{2}$; 
5 min each, twice), fixed in $4 \% \mathrm{w} / \mathrm{v}$ PFA in PBS+ $(30$ min) and quenched with $75 \mathrm{mM} \mathrm{NH}_{4} \mathrm{Cl}+0.5 \mathrm{mM} \mathrm{MgCl}_{2}$ (5 min, five times). The acini were permeabilized in $0.5 \% \mathrm{v} / \mathrm{v}$ Triton-X100 in PBS (30 min) and incubated in immunofluorescence buffer (IFB; $130 \mathrm{mM} \mathrm{NaCl}, 7 \mathrm{mM}$ $\mathrm{Na}_{2} \mathrm{HPO}_{4}, 3.5 \mathrm{mM} \mathrm{NaH}_{2} \mathrm{PO}_{4}, 7.7 \mathrm{mM} \mathrm{NaN}_{3}, 0.1 \% \mathrm{w} / \mathrm{v}$ BSA, $0.5 \% \mathrm{v} / \mathrm{v}$ Triton X-100 and $0.05 \%$ v/v Tween-20), complemented with $20 \%$ v/v non-immune goat serum (ab7481, Abcam) on a shaking platform $(2 \mathrm{~h})$. The cultures were incubated at $4^{\circ} \mathrm{C}(16 \mathrm{~h})$ with primary antibodies diluted in IFB at the following dilutions: 1/500 mouse anti-pentosidine, clone PEN-12 mAb (KH012, TransGenic Inc.), 1/250 rabbit anti-human collagen IV polyclonal antibody (pAb; R1041, Acris Antibodies), 1/250 rabbit anti-human laminin pAb (sc-5583, Santa Cruz), 1/250 rabbit anti-human E-cadherin pAb (3195, Invitrogen), 1/250 mouse anti-GM130 mAb clone EP892Y (ab52649; Abcam), 1/100 mouse anti-EEA1 mAb clone 14/EEA1 (610457, BD Transduction Laboratories) and Endo180 mAb (clones A5/158 and 39.10) [12,15,16] and washed in IFB at room temperature $(10 \mathrm{~min}$, three times). AlexaFluor goat anti-rabbit or anti-mouse IgG $(\mathrm{H}+\mathrm{L})$ secondary antibody conjugates were diluted $1 / 500$ in IFB, added to cultures and incubated at room temperature $(2 \mathrm{~h})$. Following washing in IFB and PBS+ (10 min each, three times), the acini were fixed and quenched as described above. Cell nuclei were stained with TO-PRO ${ }^{\circledR}-3$-iodide (T3605, Sigma-Aldrich) (5 min), washed with PBS+ (5 min, twice) and mounted in Vectashield ${ }^{\circledR}$ (H1000, Vector Laboratories), and epifluorescent and confocal images were acquired using a Zeiss Axiovert S100 microscope operated by MetaMorph software, and a Zeiss Confocal Axiovert 200M operated by LSM510 META software, respectively.

\section{Western blot analysis}

Immunoblotting was carried out as previously described [16], using the following primary antibody dilutions: 1/1000 anti-Endo180 clone CAT2 pAb [17], 1/1000 mouse anti-human $\beta_{1}$-integrin mAb clone $12 \mathrm{G} 10$ (MCA2028, AbD Serotec), 1/1000 anti-E-cadherin pAb, 1/10000 anti-GAPDH (ab9485, Abcam), 1/500 rabbit anti-phospho-MLC2 (Thr18/Ser19) pAb (3674S, New England Biolabs), 1/1000 mouse anti-myosin light chain (4401, New England Biolabs); followed by 1/5000 HRP-conjugated goat anti-rabbit IgG (sc-2004, Santa Cruz) or 1/500 HRP-conjugated AffiniPure goat anti-mouse IgG (H+L; 115-035-174, Jackson Immunoresearch Laboratories). Relative signal intensities were quantified from immunoblots by densitometric analysis (Image J v 1.42q) and normalized against mouse IgG-treated controls and native rBM control gels (reference for native $\mathrm{rBM}$ gels and mouse $\operatorname{IgG}=1 \mathrm{U}$ ) and adjusted for loading against GAPDH levels.

\section{Analysis of mouse tissue}

Wild-type and Endo180 ${ }^{\Delta \mathrm{Ex} 2-6 / \Delta \mathrm{Ex} 2-6}$ mice were genotyped for the Endo180 allele by Southern blot analysis
$[17,18]$. Prostate glands were excised and fixed in $4 \%$ w/v paraformaldehyde prior to processing. Sections were deparaffinized in $100 \% \mathrm{v} / \mathrm{v}$ xylene (30 min, twice) and hydrated in graded ethanol solutions (100\%, 95\%, $70 \% \mathrm{v} / \mathrm{v})$ and then double-distilled water. Antigen retrieval was performed using $20 \mu \mathrm{g} / \mathrm{ml}$ Proteinase $\mathrm{K}$ diluted in TE buffer at $37^{\circ} \mathrm{C}(30 \mathrm{~min})$, boiling in $1 \mathrm{mM}$ EDTA, pH 8 (10 min), washing in PBS (10 min, three times) and inactivation of endogenous peroxidase in $3 \% \mathrm{v} / \mathrm{v}$ hydrogen peroxidase $(10 \mathrm{~min})$. After washing in PBS (5 min, three times), the samples were incubated (1 h) in blocking solution (PBS $+20 \% \mathrm{w} / \mathrm{v}$ FBS). Sections were incubated with anti-collagen IV (R1041, Acris Antibodies) or anti-laminin (sc-5583, Santa Cruz, 1/50 in blocking solution) at $4^{\circ} \mathrm{C}(16 \mathrm{~h})$, washed in PBS (5 min, three times), incubated with AlexaFluor-488 goat anti-rabbit $\operatorname{IgG}(\mathrm{H}+\mathrm{L})$ diluted in blocking solution $(1 \mathrm{~h})$, washed in PBS (5 min, three times), counterstained with DAPI, washed with PBS (5 min, twice) and mounted in Vectashield ${ }^{\circledR}$. Images were acquired using a Zeiss S100 Axiovert epifluorescent/brightfield microscope. Densitometric analysis was performed using ImageJ algorithms to calculate the ratio of immunostained area (pixels) divided by the average intensity of background (pixels). To analyse luminal obstruction, the following assumption was made: one luminal epithelial cell layer $=$ hollow; two luminal epithelial cell layers = partial obstruction; and more than two luminal epithelial cell layers $=$ complete obstruction. Collagen IV and laminin intensities and luminal obstruction were calculated from sections of anterior and ventral prostates obtained from five wild-type and six Endo $180^{\Delta \text { Ex2-6/ } \mathrm{Ex} 2-6}$ adult (age $>18$ months) mice.

\section{Analysis of human tissue}

Tissue biopsies on the NCLPC1 and NCLPC4 prostate cancer TMAs used in this study were obtained with consent and approved by the Research Ethics Committee of Newcastle University Medical School. IHC was carried out using a Bond III fully automated staining system and Bond Polymer Refine Detection (Leica Microsystems). Antigen retrieval was carried out using Epitope retrieval, $\mathrm{pH} 6\left(100^{\circ} \mathrm{C}, 30 \mathrm{~min}\right) .1 / 2500$ rabbit anti-AGE pAb (ab23722, Abcam; performed at Imperial College London) or 1/50 anti-Endo180 (39.10; performed at Newcastle University) were applied to tissue sections (30 $\mathrm{min}$ ) and $1 \times$ diaminobenzidine was used as a chromogen. The sections were counterstained with Mayer's haematoxylin (MHS1, Sigma-Aldrich) and mounted in Faramount aqueous medium (S3025, Dako). Immunostaining was quantified as percentage positive epithelial cells $(0-100) \times$ staining intensity $(0-3)$, giving possible scores of $0-300$. Two cores were evaluated from each tumour and the mean calculated. If one core was unavailable, the value for the remaining core was used. A cut-off score of 30 defined positive versus negative tumours was used. This immunohistochemical scoring system is in routine use in the Departments of Medicine and Surgery and Cancer at Imperial College London [19]. 


\section{Statistics}

Statistical significance was evaluated using two-tailed Student's $t$-test $(\alpha=0.05)$. Kaplan-Meier analysis, log rank, Breslow and Tarone-Ware tests were conducted, using SPSS v 19.0 statistical software (SPSS Inc.). Assumption of proportional hazard was tested for all co-variates. Statistical significance was reached where $p<0.05$.

\section{Results}

Advanced glycation end-product-dependent, non-enzymatic crosslinking of the basal lamina as a model for its structural alteration during ageing

To simulate increased thickness and stiffness of the basal lamina associated with ageing, glycolaldehyde was used to non-enzymatically crosslink rBM matrix by promoting Schiff base adduct formation, Amadori rearrangement and subsequent advanced glycation end product (AGE) generation. AGE generation was determined by the measurement of fluorophores in glycolaldehyde-treated rBM [13]. Fluorophore production associated with AGE generation was effectively blocked by the Schiff base reducing agent, sodium cyanoborohydride, and the reactive aldehyde quencher, aminoguanidine (see supplementary material, Figure S1A). The formation of high molecular weight rBM protein fragments that were not able to penetrate SDS-PAGE gel (see supplementary material, Figure S1B) and structural rearrangement of the rBM major matrix components, collagen IV and laminin, into dense bundles (see supplementary material, Figure S1C, D), confirmed that glycolaldehyde treatment induced the formation of crosslinks. Rheometric analysis confirmed that there was a significant increase in the elastic moduli of rBM following its treatment with glycolaldehyde for $6 \mathrm{~h}(175 \pm 90 \mathrm{~Pa})$ or $14 \mathrm{~h}(322 \pm 160 \mathrm{~Pa})$, compared to its treatment with PBS $(120 \pm 55 \mathrm{~Pa}$ ) (see supplementary material, Figure S1E). Herein, the following nomenclature describes the different biomechanical properties of rBM after these three pretreatments: 'native' (14 h PBS); 'semi-stiff' (6 h glycolaldehyde); and 'stiff' (14 h glycolaldehyde). Importantly, the $3.2 \pm 2.0$-fold increase in elastic modulus after $14 \mathrm{~h}$ pretreatment with glycolaldehyde was concordant with the 2.5- to 3.4-fold increase in stiffness observed in malignant versus normal prostate tissue (see supplementary material, Figure S1E, Table S1) [20-25].

\section{AGE-modified basal lamina induces PEC invasiveness}

To ascertain whether basal lamina stiffness can promote tumourigenic behaviour in normal prostate epithelia, we compared three-dimensional (3D) acinar structures generated by non-transformed PECs (RWPE1 cells) in native, semi-stiff and stiff rBM. AGE generation in stiff rBM was detected by immunostaining of pentosidine, an indicator of collagen IV crosslinking [26] and a biomarker for tissue stiffness [27], and was associated with increased thickness of the collagen IV and laminin layer that assembled around PEC acini (Figure 1A). The marked depletion of collagen IV and laminin at points of PEC protrusion from the basal surface of the acini, together with mislocalization of markers of cell-cell adhesion (E-cadherin) and apical polarity (GM130; early endosomal antigen-1, EEA1) induced by glycated and crosslinked rBM (Figure 1A), suggests that a stiff microenvironment contributes to the induction of their invasive behaviour. The fact that stiffened rBM did not promote polyADP ribose polymerase (PARP) cleavage (see supplementary material, Figure S2) confirmed that the levels of AGE generation and stiffness used in our model had no effect on cell viability. In keeping with these findings, acinar morphogenesis proceeded at similar rates on native and stiff $\mathrm{rBM}$ (see supplementary material, Figure S3). Comparison of PEC acini in native and stiff rBM (Figure 2A) revealed a significant transition towards an irregular (polygonal) shape (Figure 2B), accompanied by a 0.8 -fold reduction in size (Figure 2C) and a 0.4-fold decrease in luminal:total area ratio (Figure 2D) on the latter. The respective 1.8 -fold and 2.8 -fold increases in the number of acini with PECs visibly protruding from their basal surface into semi-stiff and stiff rBM (Figure 2E) confirmed that high AGE content and stiffened basal lamina induces invasive behaviour in non-transformed PECs. These findings were validated in an alternative experimental system, in which ribose was used to non-enzymatically crosslink rBM and shown to induce similar changes in PEC acinar morphology (see supplementary material, Figure S4A-D). The promotion of PEC invasion from the basal surface of acini, breakage of the basal lamina at points of PEC protrusion, reduction in luminal area and loss of epithelial cell polarity facilitated by AGE-induced stiffness of the basal lamina suggests that this biophysical change can trigger invasive behaviour in the prostate gland.

\section{AGE-modified basal lamina induces}

\section{Endo180-dependent contractile signalling and PEC invasiveness}

In relation to the induction of PEC invasiveness by AGE-dependent basal lamina stiffness, we tested the hypothesis that CTLD2, which directly binds to glycosylated collagens, including basement membrane collagen IV [8], is a mechanotransducer that directs the response of PECs to this environmental change (Figure 1B). In support of this theory, immunostaining with a mouse anti-human Endo180 monoclonal antibody (mAb), A5/158, that recognizes CTLD2 (Figure 1B) [15], revealed that endogenously expressed Endo180 is strongly localized at the basal surface of PEC acini in native rBM (Figure 1C), where it is able to bind to extracellular glycated collagens. Immunostaining with a second mouse anti-human Endo180 mAb, 39.10, detected Endo180 exclusively in endosomes 
A

y
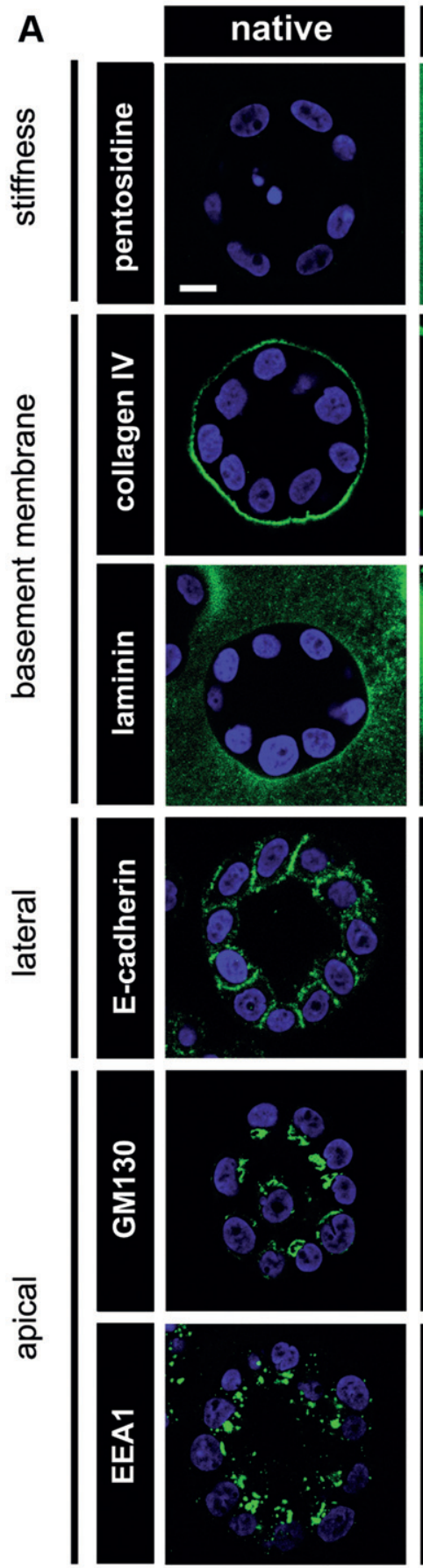
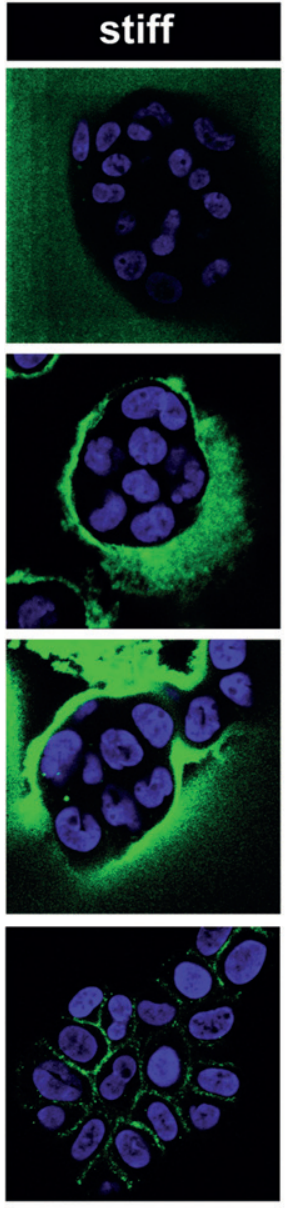
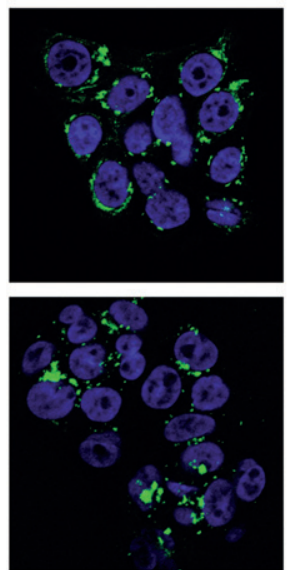

B
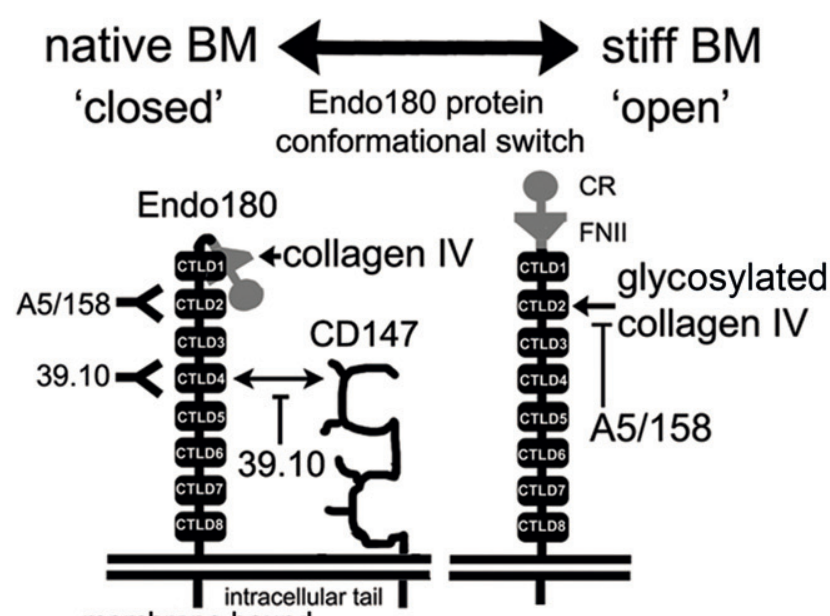

membrane-bound

Endo180-CD147

complex

$\checkmark$

Epithelial stability

internalization

into endosomes

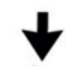

pro-invasive phenotype

C

\section{subcellular Endo180 pools}

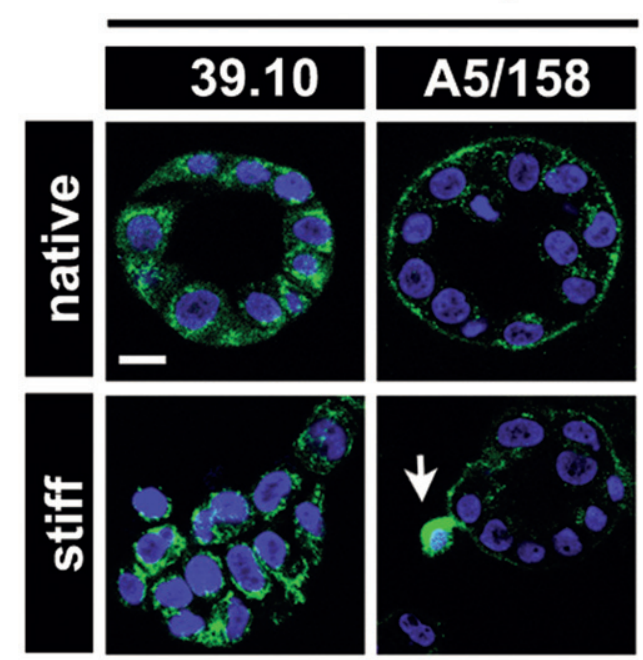

Figure 1. AGE-modified rBM affects apical-basal polarity and Endo180 subcellular localization in PEC acini. (A) Confocal microscopy images of acini grown in native or stiff rBM: TOPRO-3 nuclear stain (blue) and AGE (pentosidine), rBM matrix (collagen IV and laminin), lateral cell - cell junctions (E-cadherin) and apical polarity (GM130 and EEA1; green). (B) Glycosylated collagen IV binds to CTLD2 in Endo180 and is proposed to induce PEC invasiveness: putative 'closed' and 'open' conformations of Endo180 exist in native and stiff rBM, respectively; the ligand-binding domains in Endo180 recognized by A5/158 mAb (CTLD2) and $39.10 \mathrm{mAb}$ (CTLD4) are indicated; interaction of Endo180 with highly glycosylated CD147 at the basal plasma membrane is predicted to mask the epitope for $39.10 \mathrm{mAb}$. (C) Confocal images of the distinct subcellular localizations of Endo180, visualized using A5/158 mAb (basal) and 39.10 mAb (endosomal); arrow, invasive PEC with strong intracellular localization of Endo180; scale bars $=10 \mu \mathrm{m}$; data are representative of three independent experimental repeats.

(Figure 1C), due to its epitope CTLD4 (Figure 1B) being masked by its interaction with $\mathrm{CD} 147$ at the basal surface of PEC acini [11]. Interestingly, levels of endosomal Endo180 were visibly increased in PECs protruding from the basal surface of acini into stiff rBM (Figure 1C, arrow). Moreover, targeted blockade of CTLD2 by A5/158 mAb decreased the transition of PEC acini towards a polygonal shape (0.6-fold), decreased the basal protrusion of PECs (0.7-fold) and increased acinar size (2.2-fold) in comparison to $\operatorname{IgG}$ control treatment (Figure 3A-D). The anti-invasive effects of A5/158 mAb treatment were recapitulated in our alternative ribose-based model (see supplementary material, Figure S4E). 
A

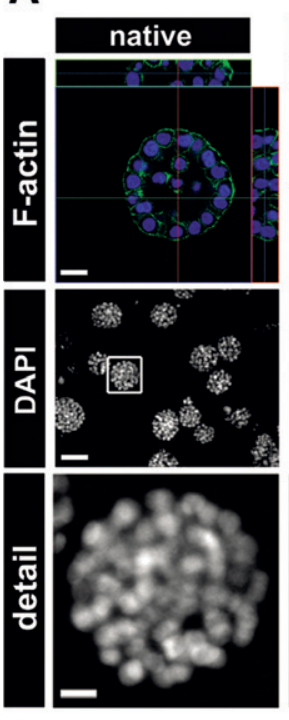

semi-stiff

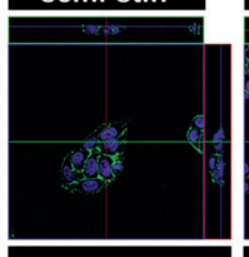

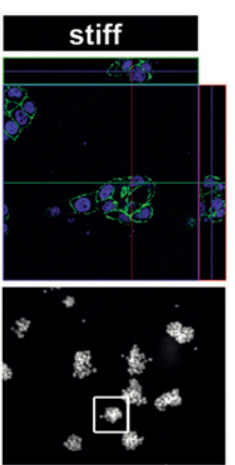
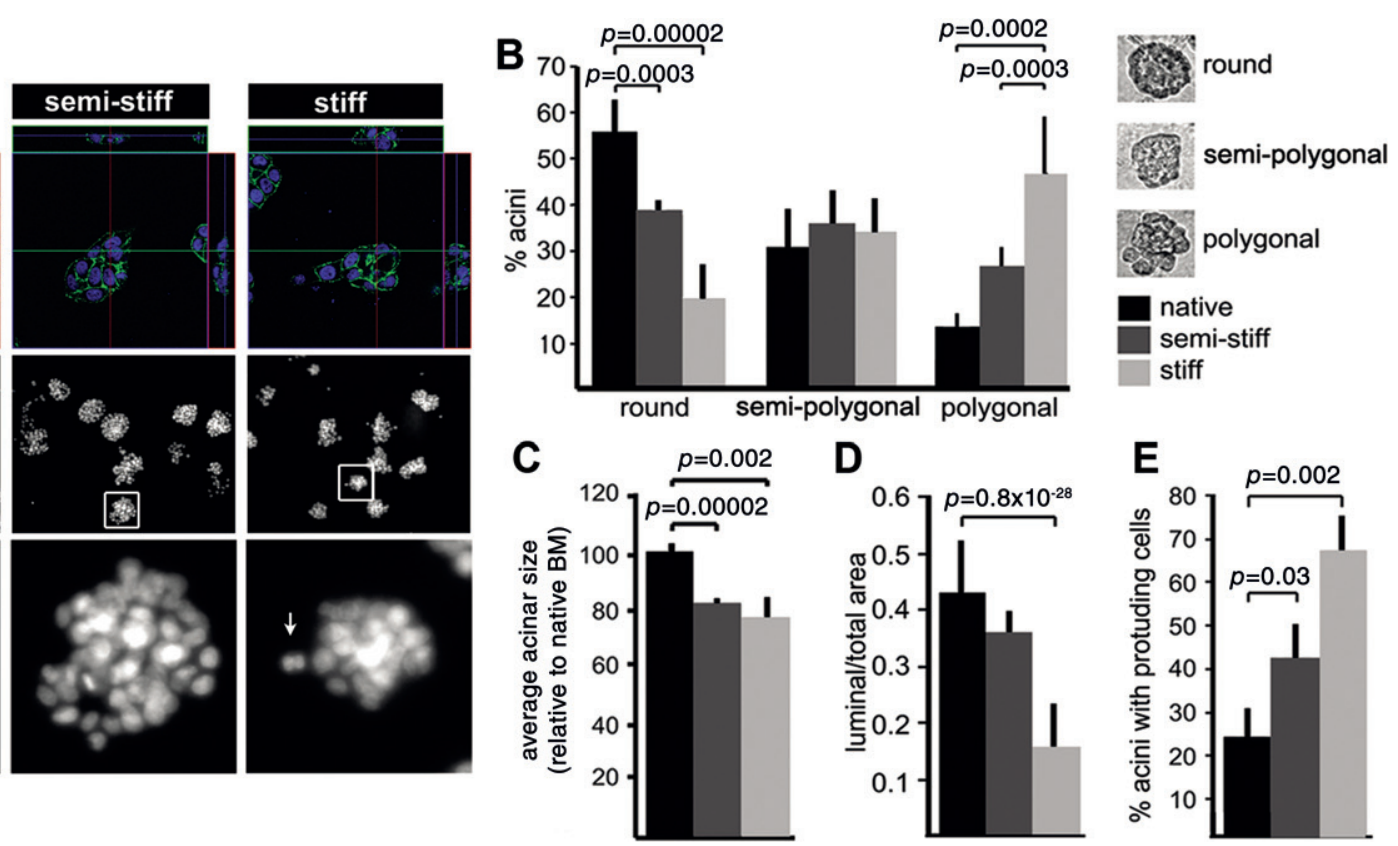

Figure 2. AGE-modified rBM induces apical and basal acinar PEC invasiveness. (A) (top) confocal microscopy images of PEC acini cultured on native, semi-stiff or stiff rBM with TOPRO-3 nuclear stain (blue) and phalloidin F-actin stain (green); scale bar=20 $\mu$ m; (middle) epifluorescent microscopy images of acini with DAPI nuclear stain; scale bar $=60 \mu \mathrm{m}$; (bottom) detail of one acinus from each condition; arrow, cell protruding from basal surface; scale bar $=10 \mu \mathrm{m}$. (B) Shape distribution of acini in native, semi-stiff and stiff rBM for 6 days (\%, mean \pm SD); graphs show $(C)$ relative acinar size (native $r B M=100 \%)$; (D) luminal:total area ratio of acini; (E) acini with cells protruding at basal surface: all graphs show values (\%, mean \pm SD for 50 acini) for native (black bars), semi-stiff (dark grey bars) and stiff (light grey bars) rBM and are representative of three independent experimental repeats.

We next considered whether the pro-invasive signals activated by stiff rBM in our 3D PEC acinar model involved the pro-migratory, actinomyosin-based, cell contractility signals generated by Endo180-containing endosomes in two dimensional (2D) culture models [12]. To explore this, pMLC2, total MLC2 and Endo180 levels were measured using immunoblot analysis of lysates generated from PEC acini cultured in native and stiff $\mathrm{rBM}$ in the presence or absence of control IgG or A5/158 mAb (Figure 4A). Stiff rBM induced a 1.3-fold increase in the pMLC2:MLC2 ratio, which was reduced to 0.3 -fold by $\mathrm{A} 5 / 158 \mathrm{mAb}$ treatment (a decrease of $\sim 77 \%$ ) without changing Endo180 expression (Figure 4B). The A5/158-sensitive (CTLD2-dependent) phosphorylation of MLC2 associated with increased rBM stiffness directly correlated with a decrease in E-cadherin and an increase in $\beta_{1}$-integrin (Figure 4B). The stiffness-induced changes in E-cadherin and $\beta_{1}$-integrin levels were restored in PEC acini by A5/158 $\mathrm{mAb}$ treatment (E-cadherin from 0.6 -fold to 1.0 -fold; $\beta_{1}$-integrin from 1.6 -fold to 0.8 -fold) (Figure 4B). These findings suggest that CTLD2-dependent actinomyosin-based contractility signals generated in PECs, at stiff basal lamina interfaces, drive their increased invasion.

\section{Endo180 modulates its extracellular matrix} remodelling partners in AGE-modified basal lamina

Given its role in collagen remodelling, we considered whether cooperative protease partners of Endo180, co-expressed on invasive PECs in human prostate tumours [10], were under the regulatory control of Endo180 under stiff conditions. Accordingly, $\mathrm{A} 5 / 158 \mathrm{mAb}$ treatment reversed the up-regulation of membrane-type 1 matrix metalloproteinase (MT1-MMP; from 1.3-fold to 0.8-fold) and urokinase plasminogen activator (uPA; from 1.2-fold to 0.5-fold) in stiff rBM (see supplementary material, Figure S5A). These findings point towards a modulatory role for Endo180 in directing pericellular proteolysis during PEC invasion through a stiffened basal lamina. However, A5/158 mAb did not inhibit collagen uptake by PECs (see supplementary material, Figure S5B), indicating that Endo180-dependent clearance of collagen degradation products is not necessary for PEC invasiveness.

\section{Prostate acini in Endo180 mutant mice have} luminal obstruction and reduced BM matrix

Our in vitro findings support the hypothesis that CTLD2 binding to modified collagen IV [8] in a stiffened basal lamina can promote PEC invasiveness (Figure 2A). To establish whether CTLD2 in Endo180 contributes to remodelling of prostate epithelia in vivo, we investigated the consequence of a targeted genetic modification in the $M R C 2$ gene that results in CTLD2 being constitutively exposed in a truncated form of the transcribed receptor (Figure 5A) [17,18]. This truncation in Endo180 simulates the 'open' conformation of the receptor that is predicted to exist in the acidic environment of endosomes (Figure 5A) [28-30]. 
A
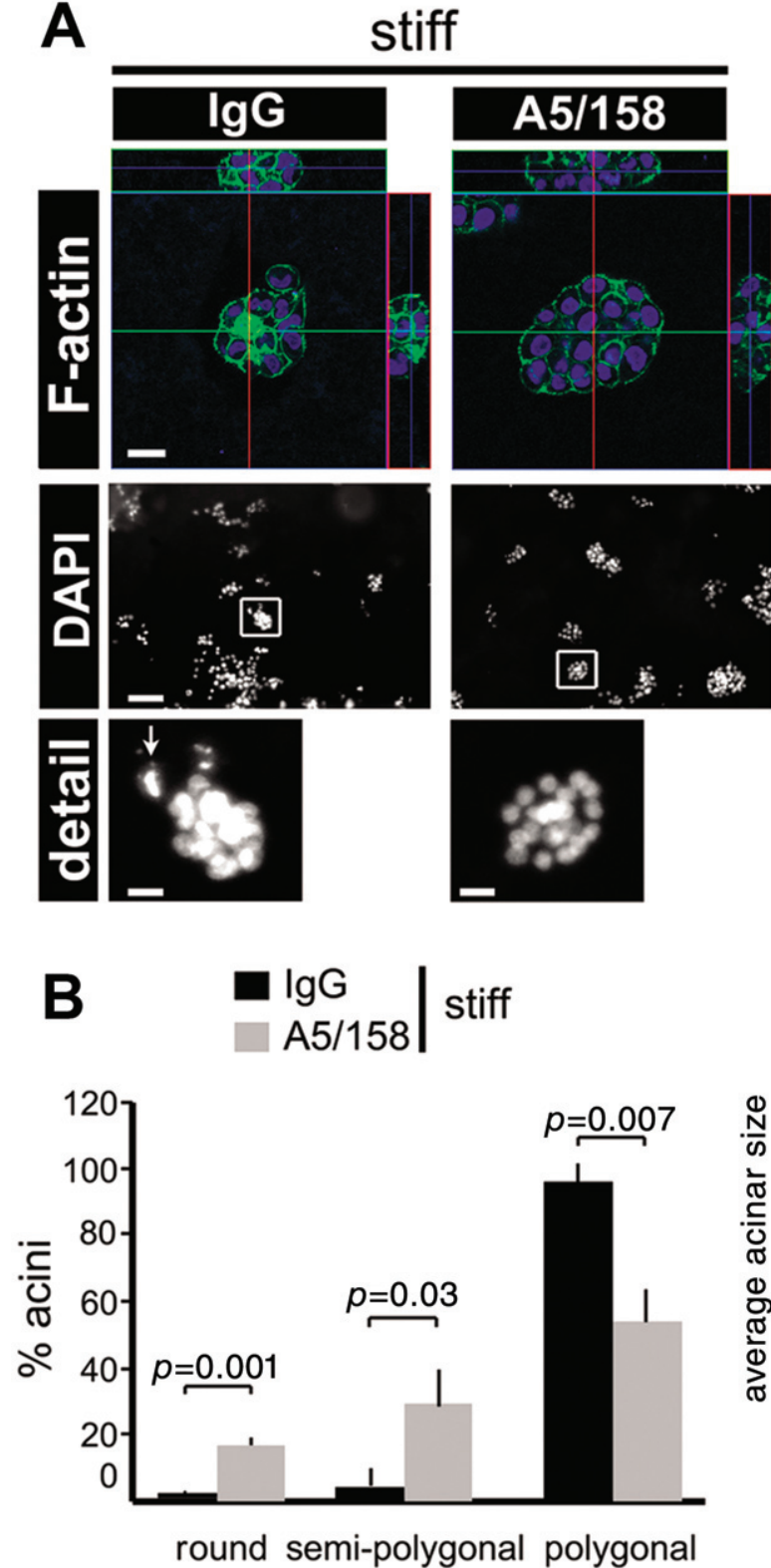

A5/158

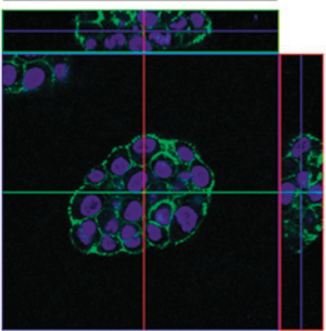

: :

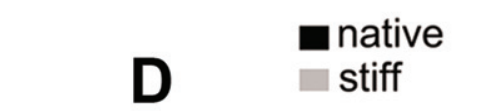

Figure 3. Endo180-dependent PEC invasion in AGE-modified rBM. (A) (top) Confocal images of acini treated with IgG or A5/158 mAb (10 $\mu \mathrm{g} / \mathrm{ml}$ ) in stiff rBM; TOPRO-3 nuclear stain (blue); phalloidin F-actin stain (green); scale bar $=20 \mu \mathrm{m}$; (middle) epifluorescent images of acini with DAPI nuclear stain; scale bar $=60 \mu \mathrm{m}$; (bottom) detail of a single acinus under each condition; arrow, cell protruding from basal surface; scale bar $=10 \mu \mathrm{m}$. Graphs show: (B) acinar shape distribution; (C) cell protrusion at the basal surface; and (D) relative acinar size (native $\mathrm{rBM}=100 \%$ ), in the presence of IgG or A5/158 in native (black bars) or stiff (light grey bars) rBM. All graphs show values (\%, mean \pm SD for 50 acini) quantified per condition and are representative of three independent experimental repeats.

Interestingly, respective reductions were observed in levels of the core basal lamina matrix components, collagen IV and laminin, in the ventral (0.7-fold and 0.6 -fold) and anterior (0.8-fold and 0.7-fold) regions of the prostate glands of Endo $180^{\Delta \mathrm{E} \times 2-6 / \Delta \mathrm{Ex} 2-6}$ mice compared to their wild-type counterparts (Figure 5B-D). Moreover, the modified basal lamina in mutant mice, with exposed CTLD2, was accompanied by an increase in complete cellular obstruction of acinar lumen in the ventral (1.7-fold) and anterior (1.9-fold) prostate, when compared to wild-type animals (Figure 5E-G), which is indicative of lesion development.

\section{Endo180 combined with AGE decreases prostate} cancer survival

AGE accumulation in human tissue is a hallmark of several age-related diseases, including multiple cancer types (reviewed in [31]). The interaction of AGE with its receptor, RAGE, is linked to the promotion of prostate cancer cell growth and invasion [32,33]; and the expression of RAGE and its ligand high-mobility group box 1 (HMBG1) has been associated with poor overall survival in a cohort of 58 patients with clinical stage III and IV prostate cancer [34]. Our in vitro findings suggest that prostate cancer has the potential to progress when the basal lamina becomes stiffened by AGE exposure and 


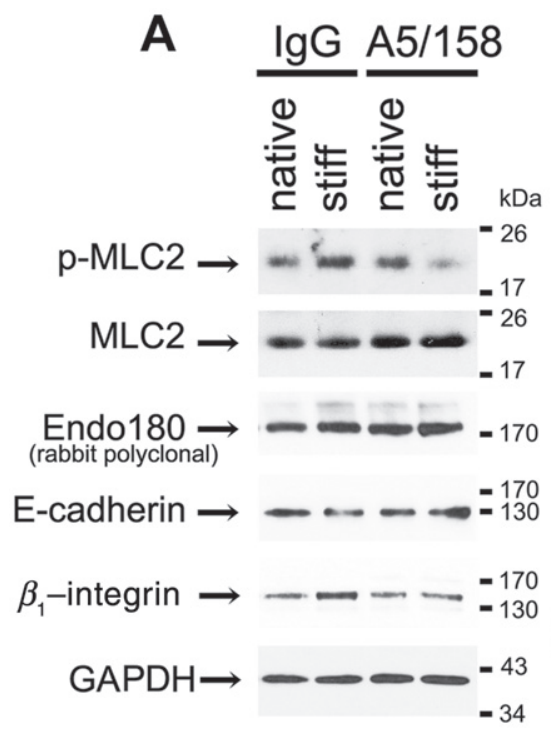

B $\square$ pMLC2 $\square$ MLC2 $\square$ Endo180

$\square$ E-cadherin $\quad \square \beta_{1}$-integrin

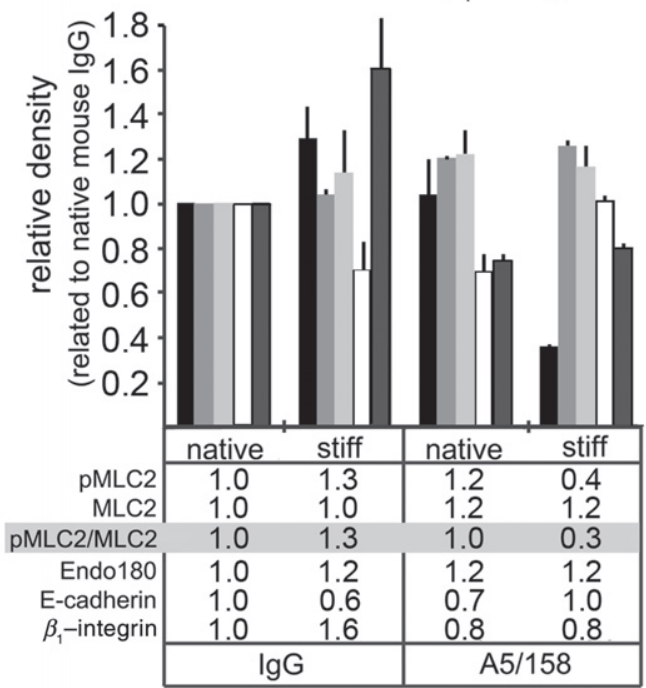

Figure 4. Endo180-dependent actinomyosin-based signal transduction in AGE-modified rBM. (A) Immunoblots show pMLC2, total MLC2, Endo180, E-cadherin, $\beta_{1}$-integrin and GAPDH levels in PEC acini cultured in native or stiff rBM in the presence of IgG or A5/158; MW markers $(\mathrm{kDa})$. (B) Graph shows relative levels of pMLC2, MLC2, pMLC2:MLC2 ratio (grey shading), Endo180, E-cadherin and $\beta_{1}$-integrin calculated from densitometric analysis of immunoblots adjusted against GAPDH loading control (native $r B M+\lg G=1$ ). All data are representative of three independent experimental repeats.

triggers Endo180-dependent mechanotransduction and invasion of PECs.

Immunostaining of two University of Newcastle tissue microarrays (NCLPC1 and NCLPC-4; see supplementary material, Table $\mathrm{S} 2$ ) revealed high levels of epithelial AGE in $55 \%(68 / 123)$ of prostate tumours (Figure 6A). Kaplan-Meier analysis revealed that high epithelial AGE content per se was not prognostic (Figure 6B), whereas high epithelial AGE accumulation combined with an Endo180-positive, but not an Endo180-negative, status (Figure 6A) was predictive of a decrease in overall survival (Figure 6C). In the subset of men with prostate tumours with high epithelial AGE content, 62\% (31/50) deaths were recorded after 5 years in the Endo180-positive group, which was significantly higher $(p<0.016)$ than the $28 \%$ (5/18) deaths recorded in the Endo180-negative subset (Figure 6C; see also supplementary material, Table S3). Endo180 had less impact on the 5-year survival rate of men with AGE-negative prostate tumours, with $69 \%$ (29/42) deaths in the Endo180-positive subset and 54\% (7/13) deaths in the Endo180-negative subset $(p=0.4)$ (Figure 6D; see also supplementary material, Table S3). This compelling evidence links the expression of Endo180 in epithelial cells, in an environment associated with increased non-enzymatic crosslinking and stiffening of the basal lamina, to the switch from indolent to aggressive prostate cancer and reduced survival.

\section{Discussion}

Here we show that the progressive thickening and stiffening of the basal lamina associated with normal ageing and metabolic disease [1-6] can induce normal PEC invasiveness. The study introduces a novel 3D model of PEC acini grown in rBM matrix that has been subject to non-enzymatic crosslinking by $\mathrm{AGE}$, recapitulating basal lamina thickening in the ageing mouse prostate, and the increased levels of tissue stiffness observed in prostate cancer (see supplementary material, Figure S1E, Table S1) [20-25]. The findings are supported by in vivo and clinical evidence that link Endo180 dysfunction to basal lamina degradation, accompanied by luminal obstruction of prostate acini and the cooperation of Endo180 with AGE in the promotion of prostate cancer progression.

In line with our previous studies, where Endo180 has been shown to play a role in EMT and prostate cancer progression $[10,11]$, and the spatiotemporal activation of actinomyosin-based contractile signals and cell migration [12], we propose a model in which two functional CTLDs in Endo180, CTLD2 and CTLD4, are responsible for directly modulating PEC invasiveness (see supplementary material, Figure S6). We believe that the molecular basis of this regulation involves a switch between the closed and open conformation of Endo180's ectodomain [28-30]. We speculate that, under normal tissue conditions, Endo180 adopts a closed conformation and maintains acinar homeostasis via the interaction of its CTLD4 with CD147 at the plasma membrane and its FNII domain with collagen IV at the basal lamina interface; whereas in stiff microenvironments it is proposed that Endo180 adopts an open conformation that allows it to sense crosslinked collagen fibres, promoting actinomyosin-based contractility and driving PEC invasion through the basal lamina. Given that antibody blockade of CTLD2 in Endo180 can partially block the 


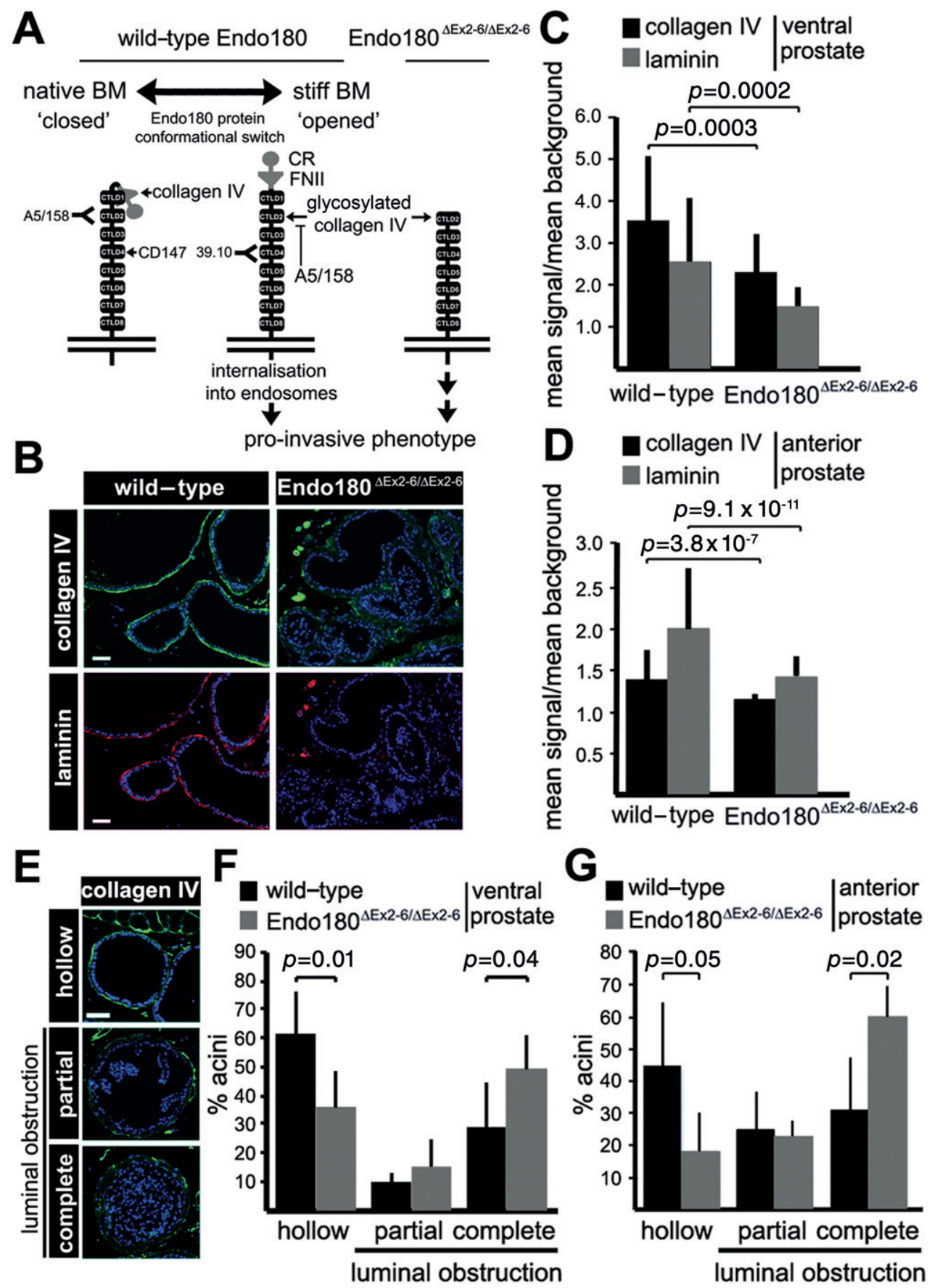

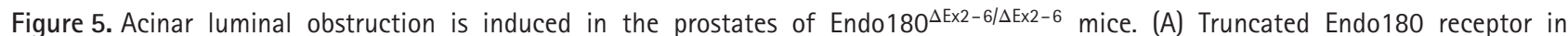
Endo $180^{\Delta \mathrm{E} \times 2-6 / \Delta \mathrm{E} \times 2-6}$ mice and proposed conformational change in Endo180 under stiff conditions. (B) Epifluorescent images of ventral prostate sections from wild-type mice and Endo $180^{\Delta \mathrm{E} \times 2-6 / \Delta \mathrm{E} \times 2-6}$ mice; TOPRO-3 nuclear stain (blue), collagen IV (green) and laminin (red): scale bar $=50 \mu \mathrm{m}$. (C) Ratio of relative staining:background intensity (mean \pm SD) for collagen IV (black bars) or laminin (grey bars) in ventral prostates of wild-type and Endo $180^{\Delta \mathrm{E} \times 2-6 / \Delta \mathrm{E} \times 2-6}$ mice. (D). Analysis in (C) conducted on anterior prostates. (E) Epifluorescent images of ventral prostate sections from wild-type and Endo $180^{\Delta \mathrm{E} \times 2-6 / \Delta \mathrm{Ex2}-6}$ mice; TOPRO-3 nuclear stain (blue), collagen IV (green); extent of luminal obstruction (hollow, partial and complete luminal obstruction); scale bar $=50 \mu \mathrm{m}$. Graphs show extent of luminal obstruction (\%, mean \pm SD for 50 acini) in ventral (F) and anterior (G) prostate glands of five wild-type mice (black bars) and six Endo $180^{\Delta \mathrm{E} \times 2-6 / \Delta \mathrm{E} \times 2-6}$ mice (grey bars).

invasive phenotype induced by the stiff basal lamina, it is plausible that this approach could be of therapeutic benefit in prostatic and other disease pathologies in which basal lamina thickness and stiffness is identified as a driving factor (see supplementary material, Figure S7).

Loss of collagen IV and laminin in the prostate gland of mutant Endo $180^{\Delta \mathrm{Ex} 2-6 / \Delta \mathrm{E} \times 2-6}$ mice indicates that the homeostasis of basal lamina turnover is defective in these animals. The mechanisms underpinning this phenomenon could involve activation of proteases that degrade the basal lamina or defective production and organization of its matrix components. The first mechanism is supported by the finding that Endo180 forms an EMT-suppressor complex with the extracellular matrix 

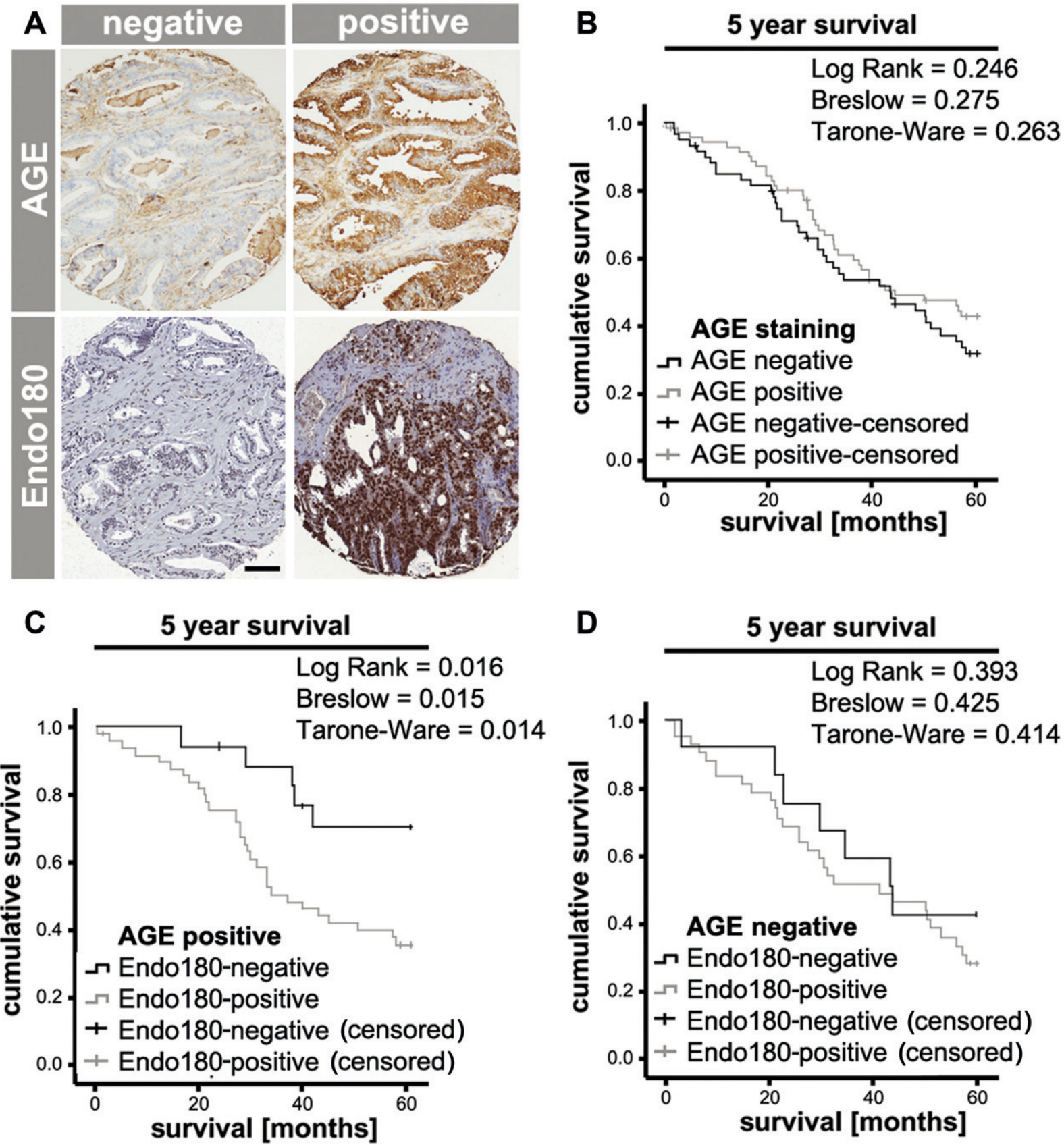

Figure 6. High epithelial AGE and Endo180 predict poor prognosis in men with prostate cancer. Immunohistochemistry of NCLPC1 and NCLPC4 tissue microarrays $(n=157)$ stained with rabbit anti-AGE pAb $(0.4 \mu \mathrm{g} / \mathrm{ml}$; brown) and Mayer's haematoxylin nuclear counterstain (blue). (A) Examples of tumour tissue cores with positive or negative epithelial AGE and Endo180 immunostaining; scale bar $=100$ $\mu \mathrm{m}$. Kaplan-Meier 5 year survival analysis of pooled data (NCLPC1 + NCLPC4) for: (B) epithelial AGE-negative (score $\leq 30$, black line) versus epithelial AGE-positive (score $\geq 30$, grey line) tumours; and epithelial Endo180-negative (score $\leq 30$, black lines) versus epithelial Endo180-positive (score $\geq 30$, grey lines) tumours in (C) epithelial AGE-positive and (D) AGE-negative patients; $p \leq 0.05$, significant for log-rank, Breslow and Tarone-Ware tests. Table S3 (see supplementary material) shows patient deaths (censored) after 3, 5, 7 and 10 years.

metalloproteinase inducer CD147 [11], and its targeted blockade results in down-regulation of MT1-MMP and uPA (see supplementary material, Figure S5), which both drive basal lamina matrix degradation [35-38]. The latter mechanism is supported from the finding in a different cellular context, whereby Endo180 orchestrates collagen deposition by primary human osteoblasts [16].

The in vitro systems used to model early tumourigenesis or tumour progression in response to tissue stiffness used constitutively active Ras transformation in a single Madine Derby canine kidney epithelial cell, or ErbB2 activation in a single MCF10A mammary gland epithelial cell, localized in corresponding non-transformed epithelial layers [39-41]. Although conceptually intriguing, these models do not explain how epithelial cancers arise when aberrant Ras or ErbB2 signalling are absent, as in the prostate gland, where mutations in either oncogene are rare $[42,43]$. The intriguing paradigm presented here suggests that 
biomechanical modification of the basal lamina is an independent trigger for PEC invasiveness.

\section{Acknowledgements}

Funded by the Association of International Cancer Research (Grant No. 08-0803), the Prostate Cancer Charity (Grant No. 110632), the Rosetrees Trust (Grant Nos M40 and M41), Tony and Rita Gallagher, and the Imperial College NHS Healthcare Trust Special Trustees. We dedicate this paper to the memory of Peter Gannaway, a good friend who passed away aged 74 years in December 2012, due to advanced prostate cancer.

\section{Author contributions}

MRT and JS conceived and designed the experiments; MRT, JHG, CB, AM, MPC, LG, SD, TC and FM performed experiments; MRT, JHG and YZZ performed data analysis; FM conducted histopathological analysis; AM provided the genotyped mouse prostate glands; JTE provided access to equipment; CR provided human tissue microarrays; JW provided funding; and MRT and JS wrote the manuscript. All authors reviewed the manuscript.

\section{References}

1. Candiello J, Balasubramani M, Schreiber EM, et al. Biomechanical properties of native basement membranes. FEBS J 2007; 274: 2897-2908.

2. Candiello J, Cole GJ, Halfter W. Age-dependent changes in the structure, composition and biophysical properties of a human basement membrane. Matrix Biol 2010; 29: 402-410.

3. Rowlatt C. Some effects of age and castration in the epithelial basal lamina of secondary sex organs in the mouse. Gerontologia 1970; 16: $182-196$.

4. Fajans SS, Williamson JR, Weissman PN, et al. Basement membrane thickening in latent diabetes. Adv Metab Disord 1973; 2: 393-399.

5. Hayden MR, Sowers JR, Tyagi SC. The central role of vascular extracellular matrix and basement membrane remodeling in metabolic syndrome and type 2 diabetes: the matrix preloaded. Cardiovasc Diabetol 2005; 4: 9.

6. Corona G, Vignozzi L, Rastrelli G, et al. Benign prostatic hyperplasia: a new metabolic disease of the aging male and its correlation with sexual dysfunctions. Int J Endocrinol 2014; 2014: Article ID 329456.

7. Wienke D, MacFadyen JR, Isacke CM. Identification and characterization of the endocytic transmembrane glycoprotein Endo180 as a novel collagen receptor. Mol Biol Cell 2003; 14: 3592-3604.

8. Jurgensen HJ, Madsen DH, Ingvarsen S, et al. A novel functional role of collagen glycosylation: interaction with the endocytic collagen receptor uPARAP/Endo180. J Biol Chem 2011; 286: 32736-32748.

9. Palmieri C, Caley MP, Purshouse K, et al. Endo180 modulation by bisphosphonates and diagnostic accuracy in metastatic breast cancer. Br J Cancer 2013; 108: 163-169.

10. Kogianni G, Walker MM, Waxman J, et al. Endo180 expression with cofunctional partners MT1-MMP and uPAR-uPA is correlated with prostate cancer progression. Eur J Cancer 2009; 45: 685-693.
11. Rodriguez-Teja M, Gronau JH, Minamidate A, et al. Survival outcome and EMT suppression mediated by a lectin domain interaction of Endo180 and CD147. Mol Cancer Res 2014; in press.

12. Sturge J, Wienke D, Isacke CM. Endosomes generate localized Rho-ROCK-MLC2-based contractile signals via Endo180 to promote adhesion disassembly. J Cell Biol 2006; 175: 337-347.

13. Kuzuya M, Satake S, Miura H, et al. Inhibition of endothelial cell differentiation on a glycosylated reconstituted basement membrane complex. Exp Cell Res 1996; 226: 336-345.

14. Debnath J, Muthuswamy SK, Brugge JS. Morphogenesis and oncogenesis of MCF-10A mammary epithelial acini grown in three-dimensional basement membrane cultures. Methods 2003; 30: 256-268.

15. Sturge J, Wienke D, East L, et al. GPI-anchored uPAR requires Endo180 for rapid directional sensing during chemotaxis. J Cell Biol 2003; 162: 789-794.

16. Caley MP, Kogianni G, Adamarek A, et al. TGF $\beta 1$-Endo180-dependent collagen deposition is dysregulated at the tumour-stromal interface in bone metastasis. J Path 2012; 226: $775-783$.

17. Sturge J, Todd SK, Kogianni G, et al. Mannose receptor regulation of macrophage cell migration. J Leukoc Biol 2007; 82: 585-593.

18. East L, McCarthy A, Wienke D, et al. A targeted deletion in the endocytic receptor gene Endo180 results in a defect in collagen uptake. ЕMBO Rep 2003; 4: 710-716.

19. Gately K, Al-Alao B, Dhillon T, et al. Overexpression of the mammalian target of rapamycin (mTOR) and angioinvasion are poor prognostic factors in early stage NSCLC: a verification study. Lung Cancer 2012; 75: 217-222.

20. Hoyt K, Castaneda B, Zhang M, et al. Tissue elasticity properties as biomarkers for prostate cancer. Cancer Biomark 2008; 4: 213-225.

21. Zhang M, Nigwekar P, Castaneda B, et al. Quantitative characterization of viscoelastic properties of human prostate correlated with histology. Ultrasound Med Biol 2008; 34: 1033-1042.

22. Carson WC, Gerling GJ, Krupski TL, et al. Material characterization of ex vivo prostate tissue via spherical indentation in the clinic. Med Eng Phys 2011; 33: 302-309.

23. Krouskop TA, Wheeler TM, Kallel F, et al. Elastic moduli of breast and prostate tissues under compression. Ultrason Imaging 1998; 20: 260-274.

24. Tuxhorn JA, Ayala GE, Smith MJ, et al. Reactive stroma in human prostate cancer: induction of myofibroblast phenotype and extracellular matrix remodeling. Clin Cancer Res 2002; 8: 2912-2923.

25. Burns-Cox N, Avery NC, Gingell JC, et al. Changes in collagen metabolism in prostate cancer: a host response that may alter progression. J Urol 2001; 166: 1698-1701.

26. Raabe HM, Molsen H, Mlinaric SM, et al. Biochemical alterations in collagen IV induced by in vitro glycation. Biochem J 1996; 319: 699-704.

27. Yoshida N, Okumura K, Aso Y. High serum pentosidine concentrations are associated with increased arterial stiffness and thickness in patients with type 2 diabetes. Metabolism 2005; 54: 345-350.

28. Boskovic J, Arnold JN, Stilion R, et al. Structural model for the mannose receptor family uncovered by electron microscopy of Endo180 and the mannose receptor. J Biol Chem 2006; 281: 8780-8787.

29. Llorca O. Extended and bent conformations of the mannose receptor family. Cell Mol Life Sci 2008; 65: 1302-1310.

30. Rivera-Calzada A, Robertson D, MacFadyen JR, et al. Three-dimensional interplay among the ligand-binding domains of the urokinase-plasminogen-activator-receptor-associated protein, Endo180. EMBO Rep 2003; 4: 807-812.

31. Nedic O, Rattan SI, Grune T, et al. Molecular effects of advanced glycation end products on cell signalling pathways, ageing and pathophysiology. Free Rad Res 2013; 47: 28-38. 
32. Ishiguro H, Nakaigawa N, Miyoshi Y, et al. Receptor for advanced glycation end products (RAGE) and its ligand, amphoterin are overexpressed and associated with prostate cancer development. Prostate 2005; 64: $92-100$.

33. Elangovan I, Thirugnanam S, Chen A, et al. Targeting receptor for advanced glycation end products (RAGE) expression induces apoptosis and inhibits prostate tumor growth. Biochem Biophys Res Comm 2012; 417: 1133-1138.

34. Zhao CB, Bao JM, Lu YJ, et al. Co-expression of RAGE and HMGB1 is associated with cancer progression and poor patient outcome of prostate cancer. Am J Cancer Res 2014; 4: 369-377.

35. Meissauer A, Kramer MD, Schirrmacher V, et al. Generation of cell surface-bound plasmin by cell-associated urokinase-type or secreted tissue-type plasminogen activator: a key event in melanoma cell invasiveness in vitro. Exp Cell Res 1992; 199: 179-190.

36. Bartolome RA, Galvez BG, Longo N, et al. Stromal cell-derived factor- $1 \alpha$ promotes melanoma cell invasion across basement membranes involving stimulation of membrane-type 1 matrix metalloproteinase and Rho GTPase activities. Cancer Res 2004; 64: 2534-2543.
37. Ueda J, Kajita M, Suenaga N, et al. Sequence-specific silencing of MT1-MMP expression suppresses tumor cell migration and invasion: importance of MT1-MMP as a therapeutic target for invasive tumors. Oncogene 2003; 22: 8716-8722.

38. Bulletti C, Jasonni VM, Polli V, et al. Basement membrane in human endometrium: possible role of proteolytic enzymes in developing hyperplasia and carcinoma. Ann NY Acad Sci 1991; 622: 376-382.

39. Hogan C, Dupre-Crochet S, Norman M, et al. Characterization of the interface between normal and transformed epithelial cells. Nat Cell Biol 2009; 11: 460-467.

40. Leung CT, Brugge JS. Outgrowth of single oncogene-expressing cells from suppressive epithelial environments. Nature 2012; 482: 410-413.

41. Levental KR, Yu H, Kass L, et al. Matrix crosslinking forces tumor progression by enhancing integrin signaling. Cell 2009; 139: $891-906$.

42. Savinainen KJ, Saramaki OR, Linja MJ, et al. Expression and gene copy number analysis of ERBB2 oncogene in prostate cancer. Am J Pathol 2002; 160: 339-345.

43. Watanabe M, Shiraishi T, Yatani R, et al. International comparison of ras gene mutations in latent prostate carcinoma. Int J Cancer 1994; 58: $174-178$.

\footnotetext{
SUPPLEMENTARY MATERIAL ON THE INTERNET

The following supplementary material may be found in the online version of this article:

Figure S1. AGE-induced non-enzymatic crosslinking and stiffness of rBM matrix

Figure S2. AGE-dependent rBM crosslinking does not affect PEC viability

Figure S3. PEC acini development on native or stiff rBM

Figure S4. Ribose-induced, AGE-dependent rBM stiffness promotes PEC invasiveness

Figure S5. Endo180-dependent up-regulation of proteases in PECs exposed to stiff rBM

Figure S6. A predicted conformational change in Endo180 induced by a stiff basal lamina

Figure S7. Schematic of how Endo180 can be targeted in stiff environments

Table S1. Stiffness of malignant compared to normal human prostate tissue

Table S2. Patient characteristics of the NCLPC1/4 prostate cancer tissue microarray

Table S3. Patient deaths associated with levels of Endo180 and AGE
}

\section{Years ago in the Journal of Pathology...}

\section{Enzyme histochemistry of gastric carcinoma}

H. T. Planteydt and R. G. J. Willighagen

To view these articles, and more, please visit: www.theiournalofpathology.com

Click 'ALL ISSUES (1892 - 2011)', to read articles going right back to Volume 1 , Issue 1.

\section{The Journal of Pathology Understanding Disease}

